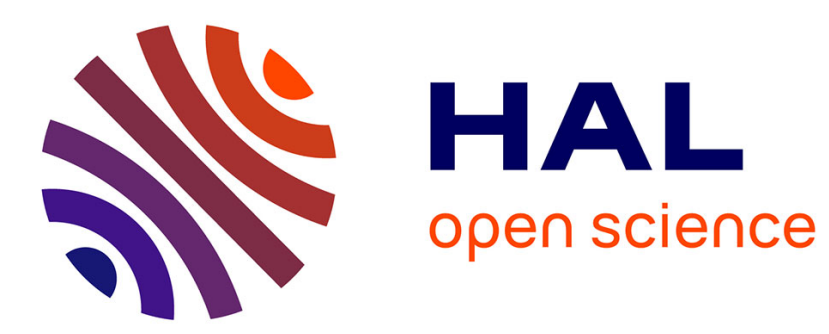

\title{
The heat kernel on noncompact symmetric spaces
}

\author{
Jean-Philippe Anker, Patrick Ostellari
}

\section{To cite this version:}

Jean-Philippe Anker, Patrick Ostellari. The heat kernel on noncompact symmetric spaces. S. G. Gindikin. Lie groups and symmetric spaces, Amer. Math. Soc., pp.27-46, 2003, Amer. Math. Soc.

Transl. Ser. 2, vol. 210. hal-00002509

\section{HAL Id: hal-00002509 \\ https://hal.science/hal-00002509}

Submitted on 9 Aug 2004

HAL is a multi-disciplinary open access archive for the deposit and dissemination of scientific research documents, whether they are published or not. The documents may come from teaching and research institutions in France or abroad, or from public or private research centers.
L'archive ouverte pluridisciplinaire HAL, est destinée au dépôt et à la diffusion de documents scientifiques de niveau recherche, publiés ou non, émanant des établissements d'enseignement et de recherche français ou étrangers, des laboratoires publics ou privés. 


\title{
THE HEAT KERNEL ON NONCOMPACT SYMMETRIC SPACES
}

\author{
JeAn-Philippe Anker \& Patrick Ostellari \\ In memory of F. I. Karpelevič (1927-2000)
}

The heat kernel plays a central role in mathematics. It occurs in several fields: analysis, geometry and - last but not least - probability theory. In this survey, we shall focus on its analytic aspects, specifically sharp bounds, in the particular setting of Riemannian symmetric spaces of noncompact type. It is a natural tribute to Karpelevič, whose pioneer work [Ka] inspired further study of the geometry of theses spaces and of the analysis of the Laplacian thereon.

This survey is based on lectures delivered by the first author in May 2002 at IHP in Paris during the Special Quarter Heat kernels, random walks $\&$ analysis on manifolds $\&$ graphs. Both authors would like to thank the organizers for their great job, as well as Martine Babillot, Gilles Carron, Sasha Grigor'yan and Jean-Pierre Otal for stimulating discussions.

\section{Preliminaries}

We shall briefly review some basics about noncompact Riemannian symmetric spaces $X=G / K$ and we shall otherwise refer to standard texbooks ([GV]; [H1], [H2], [H3]; $[\mathrm{Kn}])$ for their structure and harmonic analysis thereon.

Thus $G$ is a semisimple Lie group (real, connected, noncompact, with finite center) or more generally a reductive Lie group in the Harish-Chandra class and $K$ is a maximal compact subgroup. Let $\theta$ be the Cartan involution and let $\mathfrak{g}=\mathfrak{k} \oplus \mathfrak{p}$ be the Cartan decomposition at the Lie algebra level. $\mathfrak{g}$ is equipped with the inner product

$$
\langle X, Y\rangle=-\mathrm{B}(X, \theta Y),
$$

where $\mathrm{B}$ is the Killing form, appropriately modified if $\mathfrak{g}$ has a central component. (1.1) enables us to identify $\mathfrak{g}$ with its dual $\mathfrak{g}^{*}$, and likewise for subspaces of $\mathfrak{g}$. (1.1) induces the Riemannian structure on $X=G / K$, whose tangent space at the origin $0=e K$ is identified with $\mathfrak{p}$. Let $\mathfrak{a}$ be a Cartan subspace of $\mathfrak{p}$, let $\mathfrak{m}$ be the centralizer of $\mathfrak{a}$ in $\mathfrak{k}$ and let

$$
\mathfrak{g}=\mathfrak{a} \oplus \mathfrak{m} \oplus\left\{\oplus_{\alpha \in \Sigma} \mathfrak{g}_{\alpha}\right\}
$$

be the root space decomposition of $\mathfrak{g}$ with respect to $\mathfrak{a}$. Select in $\mathfrak{a}$ a positive Weyl chamber $\mathfrak{a}^{+}$, in $\Sigma$ the corresponding sets $\Sigma^{+}$of positive roots, $\Sigma_{0}^{+}$of positive indivisible roots, $\Pi$ of simple roots, and in $\mathfrak{g}$ the corresponding nilpotent subalgebra $\mathfrak{n}=\oplus_{\alpha \in \Sigma^{+}} \mathfrak{g}_{\alpha}$. Let $\varrho=\frac{1}{2} \sum_{\alpha \in \Sigma^{+}} m_{\alpha} \alpha$ be the half sum of positive roots, counted with multiplicities

2000 Mathematics Subject Classification. Primary 22E30, 35B50, 43A85, 58J35; Secondary 22E46, 43A80, 43A90.

Key words and phrases. Abel transform, heat kernel, maximum principle, semisimple Lie group, symmetric space, subLaplacian.

Both authors partially supported by the European Commission (IHP Network HARP) 
$m_{\alpha}=\operatorname{dim} \mathfrak{g}_{\alpha}$, let $\ell=\operatorname{dim} \mathfrak{a}$ be the rank of $X$ and let $n=\ell+\sum_{\alpha \in \Sigma^{+}} m_{\alpha}$ be the dimension of $X$. Finally $A=\exp \mathfrak{a}$ and $N=\exp \mathfrak{n}$ are closed subgroups of $G$ and $M$ denotes the centralizer of $A$ in $K$.

We shall need the following classical decompositions of $G$

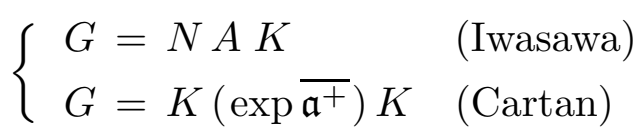

and the resulting decompositions of $X=G / K$. Let us write $x=n(x) a(x) k(x)$ in the Iwasawa decomposition, which is unique, and $A(x)=\log a(x) \in \mathfrak{a}$. The $\overline{\mathfrak{a}^{+}}$-component in the Cartan decomposition is uniquely determined, contrarily to the $K$-components. We shall write $|x|=|H|$ if $x=k_{1} e^{H} k_{2}$.

Let $\left(X_{j}\right)$ be a basis of $\mathfrak{g}$, let $b_{j k}=\mathrm{B}\left(X_{j}, X_{k}\right)$ and let $\left(b^{j k}\right)$ be the inverse matrix of $\left(b_{j k}\right)$. Then $\Omega=\sum_{j, k} b^{j k} X_{j} X_{k}$ is the Casimir element in the universal enveloping algebra $\mathcal{U}(\mathfrak{g})$. Consider in particular orthonormal bases $\left(Y_{j}\right)$ and $\left(Z_{k}\right)$ of $\mathfrak{p}$ and $\mathfrak{k}$ with respect to (1.1). Then

$$
\Omega=\Omega_{\mathfrak{p}}-\Omega_{\mathfrak{k}} \quad \text { where }\left\{\begin{array}{l}
\Omega_{\mathfrak{p}}=\sum_{j} Y_{j}^{2} \\
\Omega_{\mathfrak{k}}=\sum_{k} Z_{k}^{2}
\end{array}\right.
$$

The Laplace-Beltrami operator on $X=G / K$ can be recovered from the action of the Casimir element :

$$
\Delta f(x K)=f(x: \Omega)=f\left(x: \Omega_{\mathfrak{p}}\right) \quad \forall x \in G .
$$

Here we consider - as we shall always do - functions on $X$ as right- $K$-invariant functions on $G$ and we use the following notation for the right-action of $\mathcal{U}(\mathfrak{g})$ on $C^{\infty}(G)$ :

for all $x \in G$ and $X_{1}, \ldots, X_{r} \in \mathfrak{g}$.

$$
f\left(x: X_{1} \cdots X_{r}\right)=\left.\left.\frac{\partial}{\partial t_{1}}\right|_{t_{1}=0} \cdots \frac{\partial}{\partial t_{r}}\right|_{t_{r}=0} f\left(x e^{t_{1} X_{1}} \cdots e^{t_{r} X_{r}}\right)
$$

$L^{2}$ harmonic analysis on $X=G / K$ may be summarized by the Plancherel formula

$$
\left(\mathrm{L}_{X}, L^{2}(X)\right) \cong \int_{\mathfrak{a}^{+}}^{\oplus} \frac{d \lambda}{|\mathbf{c}(\lambda)|^{2}}\left(\pi_{\lambda}, \mathcal{H}_{\lambda}\right)
$$

which expresses the decomposition of the left regular representation of $G$ on $L^{2}(X)$ into spherical principal series. Recall that

(i) $\pi_{\lambda}$ is realized on $\mathcal{H}_{\lambda} \equiv L^{2}(K / M)$ by

$$
\left\{\pi_{\lambda}(x) \xi\right\}(k M)=e^{\left\langle\varrho+i \lambda, A\left(k^{-1} x\right)\right\rangle} \xi\left(x^{-1} \cdot k M\right) ;
$$

(ii) the Harish-Chandra $\mathbf{c}$-function, which enters the Plancherel measure, was computed explicitly by Gindikin \& Karpelevič [GK];

(iii) the decomposition (1.2) is realized by the Fourier transform and its inverse

$$
\mathcal{H} f(\lambda, k M)=\int_{G} d x f(x)\left\{\pi_{\lambda}(x) \mathbf{1}\right\}(k M)=\int_{G} d x f(x) e^{\left\langle\varrho+i \lambda, A\left(k^{-1} x\right)\right\rangle}
$$

$$
\begin{aligned}
f(x) & =\text { const. } \int_{\mathfrak{a}} \frac{d \lambda}{|\mathbf{c}(\lambda)|^{2}}\left\langle\mathcal{H} f(\lambda), \pi_{\lambda}(x) \mathbf{1}\right\rangle_{L^{2}(K / M)}= \\
& =\text { const. } \int_{\mathfrak{a}} \frac{d \lambda}{|\mathbf{c}(\lambda)|^{2}} \int_{K} d k \mathcal{H} f(\lambda, k M) e^{\left\langle\varrho-i \lambda, A\left(k^{-1} x\right)\right\rangle} .
\end{aligned}
$$

In the bi- $K$-invariant case, (1.2) boils down to

and the integral transforms (1.3) and (1.4) to

$$
L^{2}(K \backslash G / K) \cong L^{2}\left(\mathfrak{a}, \frac{d \lambda}{|\mathbf{c}(\lambda)|^{2}}\right)^{W}
$$

$$
\mathcal{H} f(\lambda)=\int_{G} d x f(x) \varphi_{\lambda}(x) \text { and } f(x)=\text { const. } \int_{\mathfrak{a}} \frac{d \lambda}{|\mathbf{c}(\lambda)|^{2}} \mathcal{H} f(\lambda) \varphi_{-\lambda}(x) .
$$

These formulas involve the spherical functions 


$$
\varphi_{\lambda}(x)=\left\langle\pi_{\lambda}(x) \mathbf{1}, \mathbf{1}\right\rangle_{L^{2}(K / M)}=\int_{K} d k e^{\langle\varrho+i \lambda, A(k x)\rangle} .
$$

The heat equation is the following parabolic evolution equation

$$
\frac{\partial}{\partial t} u(x, t)=\Delta_{x} u(x, t)
$$

with Cauchy data $u(x, 0)=f(x)$. It is solved

$$
u(x, t)=\int_{X} h_{t}(x, y) f(y) d y
$$

by the heat kernel $h_{t}(x, y)$, whose fundamental properties are recalled next.

(i) $h_{t}$ is symmetric and positive: $h_{t}(x, y)=h_{t}(y, x)>0$;

(ii) $h_{t}(x, y)$ is a smooth function of $x \in X, y \in X$ and $t \in(0,+\infty)$;

(iii) It satisfies the heat equation: $\frac{\partial}{\partial t} h_{t}(x, y)=\Delta_{x} h_{t}(x, y)=\Delta_{y} h_{t}(x, y)$;

(iv) For every $x \in X, h_{t}(x, y) d y$ is a probability measure on $X$, which converges to the Dirac measure $\delta_{x}(y)$ as $t \stackrel{>}{\longrightarrow}$.

These properties hold for quite general manifolds. In our setting, the $G$-invariance implies moreover that

(v) $h_{t}(x K, y K)=h_{t}\left(y^{-1} x\right)$ is a right convolution kernel;

(vi) $x \longmapsto h_{t}(x)$ is a bi-K-invariant function on $G$, which is thus determined by its restriction to the positive Weyl chamber.

An alternative consists in solving the heat equation (1.5) via the Fourier transform (1.3):

$$
\left\{\begin{array}{l}
\frac{\partial}{\partial t} \mathcal{H} u(\lambda, t)=-\left(|\lambda|^{2}+|\varrho|^{2}\right) \mathcal{H} u(\lambda, t) \\
\mathcal{H} u(\lambda, 0)=\mathcal{H} f(\lambda)
\end{array}\right.
$$

yields $\mathcal{H} u(\lambda, t)=\mathcal{H} f(\lambda) e^{-\left(|\lambda|^{2}+|\varrho|^{2}\right) t}$, hence $u(x, t)=\left(f * h_{t}\right)(x)$, where

$$
h_{t}(x)=\text { const. } \int_{\mathfrak{a}} \frac{d \lambda}{|\mathbf{c}(\lambda)|^{2}} e^{-t\left(|\lambda|^{2}+|\varrho|^{2}\right)} \varphi_{ \pm \lambda}(x) \text {. }
$$

\section{EXPLICIT EXPRESSIONS}

The expression (1.6) for the heat kernel, as inverse spherical Fourier transform, is neither explicit nor easy to handle in general. For instance, it is not obvious from (1.6) that $h_{t}(x)>0$. We shall now list some particular cases, where (more or less) explicit and manageable expressions are available.

\section{Case 1: $G$ complex}

This is the most elementary case, as far as spherical Fourier analysis is concerned, which boils down to Euclidean Fourier analysis on $\mathfrak{a}$ or on $\mathfrak{p}$. The following formula was written down first by Gangolli [Ga] (and must have been known to the Russian School led by Dynkin, Gelfand, Karpelevič, ... ) :

$$
h_{t}\left(e^{H}\right)=(4 \pi t)^{-\frac{n}{2}} e^{-|\varrho|^{2} t}\left\{\prod_{\alpha \in \Sigma^{+}} \frac{\langle\alpha, H\rangle}{\sinh \langle\alpha, H\rangle}\right\} e^{-\frac{|H|^{2}}{4 t}} \quad \forall t>0, \forall H \in \mathfrak{a} .
$$

Notice that the expression between braces coincides here both with the Jacobian $J(H)$ of the exponential map $\exp _{0}: \mathfrak{p} \rightarrow X$, raised to the power $-\frac{1}{2}$, and with the basic spherical function $\varphi_{0}\left(e^{H}\right)$. 
Flensted-Jensen $[\mathrm{F}]$ found a remarkable relation between spherical analysis on a real (semisimple or reductive) Lie group $G_{\mathbb{R}}$ and spherical analysis on its complexification $G_{\mathbb{C}}$, which yields the following relation between the corresponding heat kernels:

$$
h_{t}^{\mathbb{R}}\left(e^{H}\right)=\text { const. } \int_{K_{\mathbb{C}}} d k h_{\frac{t}{2}}^{\mathbb{C}}\left(k e^{2 H}\right) \quad \forall H \in \mathfrak{a} .
$$

Some information can be deduced from this formula, especially in the split case, but it has been unfortunately of little practical use in the general case so far.

All other cases rely on explicit formulas for the inverse Abel transform. Recall that the Abel transform is defined by

$$
\mathcal{A} f(H)=e^{-\langle\varrho, H\rangle} \int_{N} d n f\left(n e^{H}\right)=e^{\langle\varrho, H\rangle} \int_{N} d n f\left(e^{H} n\right) \quad \forall H \in \mathfrak{a}
$$

for bi- $K$-invariant functions $f$ on $G$, let say in the Schwartz class, and that its properties are summarized by the following commutative diagram

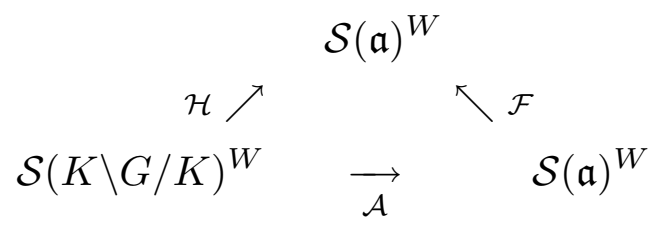

where $\mathcal{F}$ denotes the Euclidean Fourier transform and each arrow is a topological isomorphism. More information about the Abel transform and its inverse can be found in the survey [S4] by Sawyer in this volume. The diagram (2.1) yields the following abstract formula for the heat kernel

$$
h_{t}=(4 \pi t)^{-\frac{\ell}{2}} e^{-|\varrho|^{2} t} \mathcal{A}^{-1}\left\{H \longmapsto e^{-\frac{|H|^{2}}{4 t}}\right\}
$$

which can be made explicit in a few cases.

\section{Case 2: $X$ of rank one}

According to the classification, $X$ is a hyperbolic space $\mathrm{H}^{d}(\mathbb{F})$.

- $X=\mathrm{H}^{d}(\mathbb{R})$ with $d$ odd $\geq 3$ :

With an appropriate normalization of the metric,

$$
h_{t}(r)=c t^{-\frac{1}{2}} e^{-\left(\frac{d-1}{2}\right)^{2} t}\left(-\frac{1}{\sinh r} \frac{\partial}{\partial r}\right)^{\frac{d-1}{2}} e^{-\frac{r^{2}}{4 t}},
$$

where $r$ denotes the geodesic distance to the origin and $c=2^{-\frac{d+1}{2}} \pi^{-\frac{d}{2}}$.

- $X=\mathrm{H}^{d}(\mathbb{R})$ with $d$ even $\geq 2$ :

$$
h_{t}(r)=c t^{-\frac{1}{2}} e^{-\left(\frac{d-1}{2}\right)^{2} t} \int_{r}^{+\infty} \frac{d s}{\sqrt{\cosh s-\cosh r}}\left(-\frac{\partial}{\partial s}\right)\left(-\frac{1}{\sinh s} \frac{\partial}{\partial s}\right)^{\frac{d}{2}-1} e^{-\frac{s^{2}}{4 t}},
$$

where $c=2^{-\frac{d+1}{2}} \pi^{-\frac{d+1}{2}}$.

- $X=\mathrm{H}^{d}(\mathbb{C})$ with $d \geq 2$ :

where $c=2^{-d+\frac{1}{2}} \pi^{-d-\frac{1}{2}}$.

$$
h_{t}(r)=c t^{-\frac{1}{2}} e^{-d^{2} t} \int_{r}^{+\infty} \frac{d s}{\sqrt{\cosh 2 s-\cosh 2 r}}\left(-\frac{\partial}{\partial s}\right)\left(-\frac{1}{\sinh s} \frac{\partial}{\partial s}\right)^{d-1} e^{-\frac{s^{2}}{4 t}},
$$

- $X=\mathrm{H}^{d}(\mathbb{H})$ with $d \geq 2$ :

$$
\begin{aligned}
h_{t}(r) & =c t^{-\frac{1}{2}} e^{-(2 d+1)^{2} t} \times \\
& \times \int_{r}^{+\infty} \frac{d s}{\sqrt{\cosh 2 s-\cosh 2 r}}\left(-\frac{\partial}{\partial s}\right)\left(-\frac{1}{\sinh 2 s} \frac{\partial}{\partial s}\right)\left(-\frac{1}{\sinh s} \frac{\partial}{\partial s}\right)^{2(d-1)} e^{-\frac{s^{2}}{4 t}},
\end{aligned}
$$

where $c=2^{-2 d+\frac{3}{2}} \pi^{-2 d-\frac{1}{2}}$. 
- $X=\mathrm{H}^{2}(\mathbb{O})$ :

$$
\begin{aligned}
h_{t}(r) & =c t^{-\frac{1}{2}} e^{-11^{2} t} \times \\
& \times \int_{r}^{+\infty} \frac{d s}{\sqrt{\cosh 2 s-\cosh 2 r}}\left(-\frac{\partial}{\partial s}\right)\left(-\frac{1}{\sinh 2 s} \frac{\partial}{\partial s}\right)^{3}\left(-\frac{1}{\sinh s} \frac{\partial}{\partial s}\right)^{4} e^{-\frac{s^{2}}{4 t}},
\end{aligned}
$$

where $c=2^{-\frac{9}{2}} \pi^{-\frac{17}{2}}$.

These formulas are constantly rediscovered, which makes it hard to give a fair historical account. The simplest case is $\mathrm{H}^{3}(\mathbb{R})$, which corresponds to the complex group $G=$ $\mathrm{SL}(2, \mathbb{C})$. The case $\mathrm{H}^{2}(\mathbb{R})$ is usually attributed to McKean $[\mathrm{Mc}]$, although the Abel transform and its inverse had been considered earlier for $G=\operatorname{SL}(2, \mathbb{R})$, in connection with the Selberg trace formula [Go]. The difference between $d$ odd and $d$ even in the real case is only apparent. Up to constants, the family of Abel transforms on $\mathrm{H}^{d}(\mathbb{R})$ occurs indeed discretely in the one-parameter group of Weyl fractional transforms $\left\{\mathcal{W}_{\mu}^{\tau}\right\}_{\mu \in \mathbb{C}}$ with $\tau=1$, where

$$
\mathcal{W}_{\mu}^{\tau} f(r)=\left\{\begin{array}{cl}
\frac{1}{\Gamma(\mu)} \int_{r}^{+\infty} d(\cosh \tau s)(\cosh \tau s-\cosh \tau r)^{\mu-1} f(s) & \text { if } \operatorname{Re} \mu>0 \\
\left(-\frac{\partial}{\partial(\cosh \tau r)}\right) f(r) & \text { if } \quad \mu=-1
\end{array}\right.
$$

Hence (2.2) and (2.3) rewrite

$$
h_{t}^{(d)}(r)=(2 \pi)^{-\frac{d-1}{2}} e^{-\left(\frac{d-1}{2}\right)^{2} t} \mathcal{W}_{-\frac{d-1}{2}}^{1}\left(\frac{1}{\sqrt{4 \pi t}} e^{-\frac{r^{2}}{4 t}}\right) .
$$

This explains in particular the following recurrence, which was observed first by Millson, according to $[\mathrm{DGM}]$ :

$$
h_{t}^{(d+2)}(r)=\frac{1}{2 \pi} e^{-d t}\left(-\frac{1}{\sinh r} \frac{\partial}{\partial r}\right) h_{t}^{(d)}(r),
$$

The heat kernel on all hyperbolic spaces $\mathrm{H}^{d}(\mathbb{F})$ was first written down by Lohoué \& Rychener [LRy]. The Abel transform and its inverse had been computed earlier [Ko1] within the framework of Jacobi function theory developed by Flensted-Jensen \& Koornwinder. The general expression for $\mathrm{H}^{d}(\mathbb{C}), \mathrm{H}^{d}(\mathbb{H})$ and $\mathrm{H}^{2}(\mathbb{O})$ involves two Weyl fractional transforms parametrized by the root multiplicities:

$$
h_{t}(r)=c t^{-\frac{1}{2}} e^{-\left(\frac{m_{1}}{2}+m_{2}\right)^{2} t}\left(\mathcal{W}_{-\frac{m_{2}}{2}}^{2} \circ \mathcal{W}_{-\frac{m_{1}}{2}}^{1}\right)\left(e^{-\frac{r^{2}}{4 t}}\right)
$$

where $c=2^{-\frac{m_{1}+1}{2}} \pi^{-\frac{m_{1}+m_{2}+1}{2}}$. This formula extends straightforwardly to Damek-Ricci spaces [ADY], up to renormalization. Among other approaches, let us point out the work of Gruet $[\mathrm{Gr}]$ who reobtains the case $\mathrm{H}^{d}(\mathbb{R})$ by specializing a stochastic integral initially established by Yor in connection with financial mathematics.

Case 3: $G=\mathrm{SU}(p, q)$ with $p \leq q$

In appropriate coordinates,

$$
\begin{aligned}
& h_{t}\left(e^{r_{1} H_{1}+\ldots+r_{p} H_{p}}\right)= \\
& =\text { const. } t^{-\frac{p}{2}} e^{-|\varrho|^{2} t}\left\{\prod_{1 \leq i<j \leq p} \sinh \left(r_{i}+r_{j}\right) \sinh \left(r_{i}-r_{j}\right)\right\}^{-1} \times \\
& \times \int_{r_{1}}^{+\infty} \frac{d s_{1}}{\sqrt{\cosh 2 s_{1}-\cosh 2 r_{1}}}\left(-\frac{\partial}{\partial s_{1}}\right)\left(-\frac{1}{\sinh s_{1}} \frac{\partial}{\partial s_{1}}\right)^{q-p} \times \ldots \\
& \ldots \times \int_{r_{p}}^{+\infty} \frac{d s_{p}}{\sqrt{\cosh 2 s_{p}-\cosh 2 r_{p}}}\left(-\frac{\partial}{\partial s_{p}}\right)\left(-\frac{1}{\sinh s_{p}} \frac{\partial}{\partial s_{p}}\right)^{q-p} \times \\
& \times\left\{\prod_{1 \leq i<j \leq p}\left(\frac{\partial}{\partial s_{i}}+\frac{\partial}{\partial s_{j}}\right)\left(\frac{\partial}{\partial s_{i}}-\frac{\partial}{\partial s_{j}}\right)\right\} e^{-\frac{|s|^{2}}{4 t} .} .
\end{aligned}
$$


This expression is a mixture of the complex and the rank one cases. It is deduced from an explicit formula for the spherical functions (hence for the Abel transform and its inverse), which is due to Berezin \& Karpelevič [BK]. See [A2] and the references cited therein.

Case 4: $G=\mathrm{SL}(d, \mathbb{F})$

In the nineties, Sawyer has produced explicit and manageable formulas for the inverse Abel transform on all symmetric spaces $X=G / K$ with root system of type $A$, which correspond to $G=\operatorname{SL}(d, \mathbb{R}), \operatorname{SL}(d, \mathbb{C}), \operatorname{SL}(d, \mathbb{H})$ or $\operatorname{SL}(3, \mathbb{O})$. They involve a mixture of integral and differential operators in the first case, and purely differential operators in the last three cases. We shall not reproduce his formulas here, but refer instead to the original articles ([S1], [S2], [S3]) and to his contribution [S4] to this volume.

\section{Estimates}

In this section we first state and comment a global estimate for the heat kernel on $X=G / K$ (same upper and lower bound, up to constants) that was conjectured by the first author and that we have recently established [AO]. Next we present our proof in the simplest case, namely real hyperbolic spaces $X=\mathrm{H}^{n}(\mathbb{R})$. The symbol $\asymp$ between two positive expressions means that their ratio is bounded above and below.

Main Theorem: We have

$$
h_{t}\left(e^{H}\right) \asymp t^{-\frac{n}{2}}\left\{\prod_{\alpha \in \Sigma_{0}^{+}}(1+\langle\alpha, H\rangle)(1+t+\langle\alpha, H\rangle)^{\frac{m_{\alpha}+m_{2 \alpha}}{2}-1}\right\} e^{-|\varrho|^{2} t-\langle\varrho, H\rangle-\frac{|H|^{2}}{4 t}}
$$

for all $t>0$ and $H \in \overline{\mathfrak{a}^{+}}$.

The upper bound was conjectured in [A2] on the basis of some particular cases (see section 1) where explicit expressions were available. Independently and around the same time, Davies \& Mandouvalos $[\mathrm{DM}]$ established the estimate (3.1) for $X=\mathrm{H}^{n}(\mathbb{R})$. This result was extended to all hyperbolic spaces in [GM], to Damek-Ricci spaces in [ADY], and to more general Sturm-Liouville operators on the half-line $[0,+\infty)$ in [LRo]. In a series of papers ([S1], [S2], [S3]), Sawyer established the upper bound for all groups $G=\mathrm{SL}(n, \mathbb{F})$ and showed that it was a lower bound too for $G=\operatorname{SL}(3, \mathbb{R})$. This led the first author to conjecture that he had been lucky enough to guess the correct upper and lower bound. In joint works ([AJ1], [AJ2]) with Ji, he established (3.1) when $|H|=\mathcal{O}(1+t)$ (and also for all $t>0$ provided $H$ stays far away from the walls of $\left.\overline{\mathfrak{a}^{+}}\right)$. This is arguably the most important range, since heat diffuses essentially with finite speed on $X$ (see subsection 4.1). But it misses typically fixed time estimates. On the other hand, our present method yields only bounds, while the analysis carried out in [AJ1] yields also asymptotics and estimates for derivatives.

Let us comment on the right hand side of (3.1). The power $t^{-n / 2}$ and the Gaussian $e^{-|H|^{2} /(4 t)}$ are expected from the Euclidean case $X=\mathbb{R}^{n}$, where the heat kernel writes $h_{t}^{\text {eucl }}(r)=(4 \pi t)^{-n / 2} e^{-r^{2} /(4 t)}$. The spectral gap is responsible for the exponential $e^{-|\varrho|^{2} t}$ ( recall that the $L^{2}$ spectrum of $\Delta$ is equal to the half line $\left(-\infty,-|\varrho|^{2}\right]$ ). In order to understand the remaining factors, let us split the range $\overline{\mathfrak{a}^{+}} \times(0,+\infty)$ of the space-time variable $(H, t)$, depending whether the various coordinates $\langle\alpha, H\rangle(\alpha \in \Pi)$ are larger or smaller than $t$, and let us start with the main two cases :

- Assume that $t=\mathcal{O}\left(1+\min _{\alpha \in \Pi}\langle\alpha, H\rangle\right)$, for instance $t$ is bounded above. Then the right hand side of (3.1) is comparable to the expression $t^{-n / 2} J(H)^{-1 / 2} e^{-|\varrho|^{2} t-|H|^{2} /(4 t)}$, where 


$$
J(H)=\operatorname{det}\left(\left.\frac{\sinh \operatorname{ad} H}{\operatorname{ad} H}\right|_{\mathfrak{p}}\right)=\prod_{\alpha \in \Sigma^{+}}\left(\frac{\sinh \langle\alpha, H\rangle}{\langle\alpha, H\rangle}\right)^{m_{\alpha}}
$$

is the Jacobian of the exponential map $\exp _{0}: \mathfrak{p} \rightarrow X$.

- Assume that $t$ is bounded below and that $|H|=\mathcal{O}(t)$. Then the right hand side of (3.1) is comparable to $t^{-\ell / 2-\left|\Sigma_{0}^{+}\right|} \varphi_{0}\left(e^{H}\right) e^{-|\varrho|^{2} t-|H|^{2} /(4 t)}$. This expression involves the basic spherical function $\varphi_{0}$, which is a distinguished ground state of $\Delta$ on $X$ (the unique $K$-invariant one, up to constants). Its exact behavior was determined in [A1], improving slightly upon Harish-Chandra's estimates :

$$
\varphi_{0}\left(e^{H}\right) \asymp\left\{\prod_{\alpha \in \Sigma_{0}^{+}}(1+\langle\alpha, H\rangle)\right\} e^{-\langle\varrho, H\rangle} \quad \forall H \in \overline{\mathfrak{a}^{+}} .
$$

The intermediate cases combine these two extreme cases. Assume that $t$ is bounded below and that

$$
\begin{cases}\langle\alpha, H\rangle \leq t & \forall \alpha \in I \\ \langle\alpha, H\rangle \geq t \quad \forall \alpha \in \Pi \backslash I\end{cases}
$$

where $I$ is a proper subset of $\Pi$. Then the right hand side of (3.1) is comparable to $t^{-\frac{\ell}{2}-\left|\Sigma_{I, 0}^{+}\right|-\frac{m^{I}}{2}} \varphi_{I, 0}\left(e^{H}\right) J^{I}(H)^{-\frac{1}{2}} e^{-|\varrho|^{2} t-\frac{|H|^{2}}{4 t}}$.

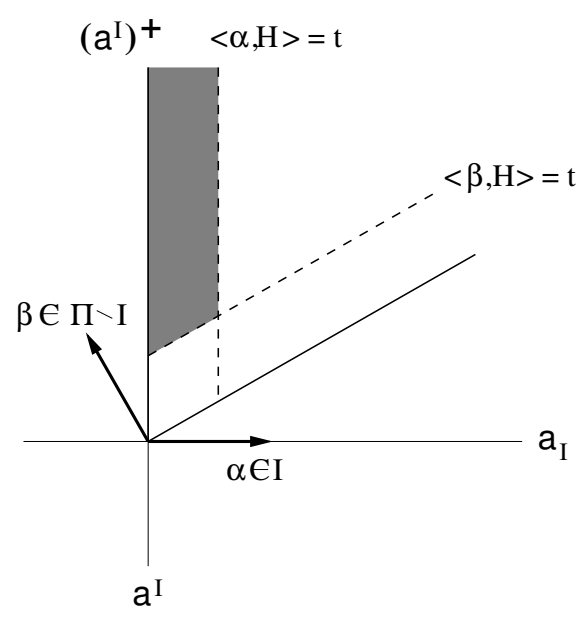

Let us give a hint of our notation, which is rather standard in this setting (except for a possible interchange of the lower and the upper index $I$ ). Subsets $I \subset \Pi$ are in 1-to-1 correspondence with Weyl chamber faces

$$
\left(\mathfrak{a}^{I}\right)^{+}=\left\{H \in \overline{\mathfrak{a}^{+}} \mid\langle\alpha, H\rangle=0 \forall \alpha \in I,\langle\beta, H\rangle>0 \forall \beta \in \Pi \backslash I\right\}
$$

and with standard parabolic subgroups $P^{I}=G_{I} A^{I} N^{I}$. As the reader may have guessed, the lower index will be used for quantities attached to $I$ and the upper index for complementary quantities. For instance, $\Sigma_{I}$ denotes the root subsytem generated by $I$ and $\varphi_{I, 0}$ the basic spherical function of the symmetric subspace $X_{I}=G_{I} / K_{I}$, while $m^{I}=\sum_{\alpha \in \Sigma^{+} \backslash \Sigma_{I}^{+}} m_{\alpha}=\operatorname{dim} N^{I}$ and $J^{I}(H)=\frac{J(H)}{J_{I}(H)}=\prod_{\alpha \in \Sigma^{+} \backslash \Sigma_{I}^{+}}\left(\frac{\sinh \langle\alpha, H\rangle}{\langle\alpha, H\rangle}\right)^{m_{\alpha}}$.

Our proof of (3.1) may look involved, especially in higher rank, but the underlying idea is quite simple. Let us denote by $\mathrm{D}=\frac{\partial}{\partial t}-\Delta_{x}$ the heat operator on $X \times \mathbb{R}$. Since the right hand side of (3.1) is comparable to a fundamental solution, it should be almost killed by $\mathrm{D}$ on $X \times(0,+\infty)$. Appropriate corrections yield supersolutions, resp. subsolutions, which satisfy the parabolic minimum, resp. maximum principle. Getting this way heat kernel estimates is not a new idea. It was used for instance by Yau and his collaborators in the Riemannian setting (see [C]) and must go back beyond. 
Proposition (weak parabolic minimum principle): Let $\Omega$ be a domain (i.e. a connected open subset with compact closure) in a Riemannian manifold $X$ and let $t_{0}<t_{1}$ be two real numbers. Assume that $u \in C^{2,1}\left(\Omega \times\left(t_{0}, t_{1}\right]\right) \cap C^{0}\left(\bar{\Omega} \times\left[t_{0}, t_{1}\right]\right)$ satisfies

(i) $\mathrm{D} u \geq 0$ in $\Omega \times\left(t_{0}, t_{1}\right]$ (supersolution),

(ii) $u \geq 0$ on the boundary components $\Omega \times\left\{t_{0}\right\}$ and $\partial \Omega \times\left[t_{0}, t_{1}\right]$.

Then $u \geq 0$ throughout $\bar{\Omega} \times\left[t_{0}, t_{1}\right]$.

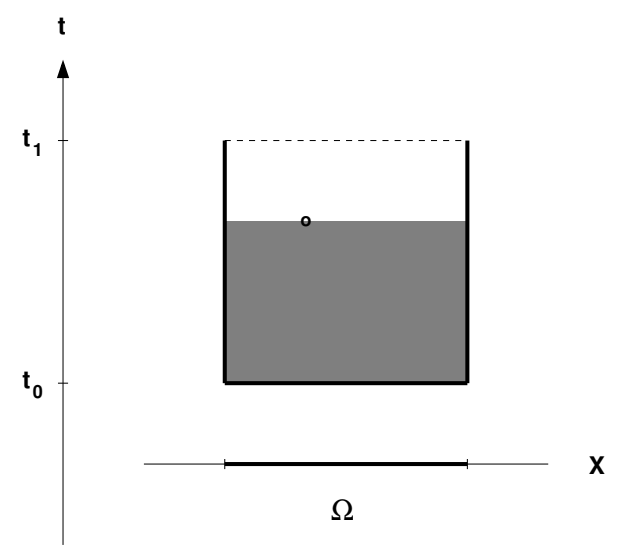

This principle holds actually for more general domains in $X \times \mathbb{R}$. We refer to [PW] for a proof, which extends straightforwardly to our setting, as well as for the strong parabolic minimum principle, which asserts that $u=0$ throughout $\bar{\Omega} \times\left[t_{0}, t_{*}\right]$ provided $u\left(x_{*}, t_{*}\right)=0$ at some point $\left(x_{*}, t_{*}\right) \in \Omega \times\left(t_{0}, t_{1}\right]$.

We shall now specialize our proof to the case $X=\mathrm{H}^{n}(\mathbb{R})$ and thus reprove differently the estimate

$$
h_{t}(r) \asymp t^{-\frac{n}{2}}(1+r)(1+r+t)^{\frac{n-3}{2}} e^{-\left(\frac{n-1}{2}\right)^{2} t-\frac{n-1}{2} r-\frac{r^{2}}{4 t}} \quad \forall t>0, \forall r \geq 0,
$$

which is due initially to Davies \& Mandouvalos $[\mathrm{DM}]$ in this setting. As usual, $r$ denotes the geodesic distance to the origin. Although the argument will be always the same, carrying out details requires to work separately in the following three regions

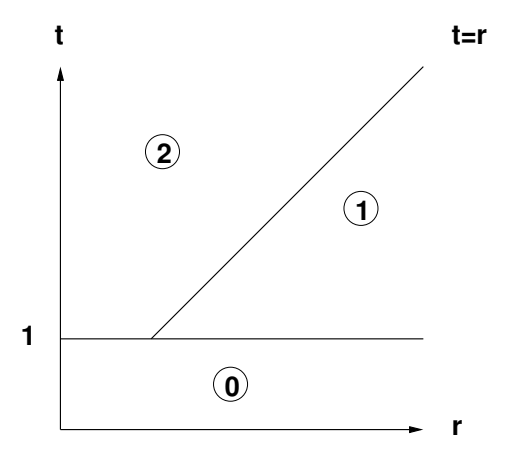

Region 0 : Let us establish (3.3), assuming that $t$ is bounded above, let say $0<t \leq 1$. We shall compare the hyperbolic heat kernel $h_{t}^{\text {hyp }}(r)=h_{t}(r)$ with the Euclidean heat kernel $h_{t}^{\text {eucl }}(r)=(4 \pi t)^{-\frac{n}{2}} e^{-\frac{r^{2}}{4 t}}$. For this purpose, let us recall the expression of the radial part of the Euclidean Laplacian and its hyperbolic counterpart:

$$
\operatorname{rad} \Delta_{\text {eucl }}=\frac{\partial^{2}}{\partial r^{2}}+\frac{n-1}{r} \frac{\partial}{\partial r}=\frac{\partial^{2}}{\partial r^{2}}+\frac{\delta_{\text {eucl }}^{\prime}}{\delta_{\text {eucl }}} \frac{\partial}{\partial r}=\delta_{\text {eucl }}^{-\frac{1}{2}} \circ \frac{\partial^{2}}{\partial r^{2}} \circ \delta_{\text {eucl }}^{\frac{1}{2}}-\omega_{\text {eucl }},
$$

where $\delta_{\text {eucl }}=c_{n} r^{n-1}$ is the volume density and $\omega_{\text {eucl }}=\delta_{\text {eucl }}^{-\frac{1}{2}} \frac{\partial^{2}}{\partial r^{2}} \delta_{\text {eucl }}^{\frac{1}{2}}=\frac{n-1}{2} \frac{n-3}{2} r^{-2}$. Similarly,

$$
\operatorname{rad} \Delta_{\text {hyp }}=\frac{\partial^{2}}{\partial r^{2}}+(n-1) \operatorname{coth} r \frac{\partial}{\partial r}=\frac{\partial^{2}}{\partial r^{2}}+\frac{\delta_{\text {hyp }}^{\prime}}{\delta_{\text {hyp }}} \frac{\partial}{\partial r}=\delta_{\text {hyp }}^{-\frac{1}{2}} \circ \frac{\partial^{2}}{\partial r^{2}} \circ \delta_{\text {hyp }}^{\frac{1}{2}}-\omega_{\text {hyp }},
$$


where $\delta_{\text {hyp }}=c_{n}(\sinh r)^{n-1}$ and $\omega_{\text {hyp }}=\delta_{\text {hyp }}^{-\frac{1}{2}} \frac{\partial^{2}}{\partial r^{2}} \delta_{\text {hyp }}^{\frac{1}{2}}=\left(\frac{n-1}{2}\right)^{2}+\frac{n-1}{2} \frac{n-3}{2}(\sinh r)^{-2}$. In the present region, (3.3) amounts to

$$
h_{t}^{\text {hyp }}(r) \asymp J(r)^{-\frac{1}{2}} h_{t}^{\text {eucl }}(r),
$$

where $J=\frac{\delta_{\text {hyp }}}{\delta_{\text {eucl }}}=\left(\frac{\sinh r}{r}\right)^{n-1}$ is the Jacobian of the exponential map. Let us denote by $h_{0}(r, t)$ the right hand side of (3.4) and let us compute

$$
\frac{\mathrm{D}_{\text {hyp }} h_{0}}{h_{0}}=\frac{\mathrm{D}_{\text {eucl }} h_{t}^{\text {eucl }}}{h_{t}^{\text {eucl }}}+\omega_{\text {hyp }}-\omega_{\text {eucl }}=\left(\frac{n-1}{2}\right)^{2}-\frac{n-1}{2} \frac{n-3}{2}\left(\frac{1}{r^{2}}-\frac{1}{\sinh ^{2} r}\right) .
$$

This expression is clearly bounded. Consider the hyperbolic heat kernel $h_{R, t}^{\text {hyp }}(r)$ in the ball $\Omega_{R} \subset X$ of radius $R>0$ centered at the origin, with Dirichlet boundary condition $h_{R, t}^{\text {hyp }}(R)=0$. It is well known that $h_{R, t}^{\text {hyp }}(r) \nearrow h_{t}^{\text {hyp }}(r)$ as $R \nearrow+\infty$. Let us apply the minimum principle in $\Omega_{R} \times[0,1]$ to the expression

Since

$$
u(r, t)=e^{c t} h_{0}(r, t)-h_{R, t}^{\text {hyp }}(r) .
$$

$$
\left\{\begin{array}{l}
\frac{D_{\text {hyp }} u}{e^{c t} h_{0}}=c+\frac{D_{\text {hyp }} h_{0}}{h_{0}} \geq 0 \quad \text { provided } c>0 \text { is large enough } \\
u(r, 0)=\delta_{0}(r)-\delta_{0}(r)=0 \text { and } u(R, t)=e^{c t} h_{0}(R, t) \geq 0
\end{array}\right.
$$

we have $u(r, t) \geq 0$ i.e. $h_{R, t}^{\text {hyp }}(r) \leq e^{c t} h_{0}(r, t)$ throughout $\Omega_{R} \times(0,1]$. One needs actually to be more careful in order to ensure that the supersolution $u$ is continuous as $t \searrow 0$. This can be achieved by considering initial datas which are nonnegative continuous functions supported inside $\Omega_{R}$. Letting $R \nearrow+\infty$, we obtain finally

$$
h_{t}^{\text {hyp }}(r) \leq C h_{0}(r, t) \quad \forall(r, t) \in[0,+\infty) \times(0,1] .
$$

By interchanging the Euclidean and the hyperbolic roles, one obtains the converse estimate

$$
h_{t}^{\mathrm{eucl}}(r) \leq C J(r)^{\frac{1}{2}} h_{t}^{\text {hyp }}(r)
$$

in the same way. This trick was pointed out to us by Gilles Carron.

Region 1: Let us analyze the right hand side of (3.3), assuming that $t$ is bounded below and that $t=\mathcal{O}(r)$, let say $1 \leq t \leq r$.

In this range, (3.3) amounts to

$$
h_{t}^{\text {hyp }}(r) \asymp J(r)^{-\frac{1}{2}} e^{-\left(\frac{n-1}{2}\right)^{2} t} h_{t}^{\text {eucl }}(r) .
$$

Denoting by $h_{1}(r, t)$ the right hand side of (3.6) and resuming the computation (3.5), we see that the expression

is $\mathcal{O}\left(\frac{1}{r^{2}}\right)$, hence $\mathcal{O}\left(\frac{1}{t^{2}}\right) . \frac{\mathrm{D}_{\text {hyp }} h_{1}}{h_{1}}=-\frac{n-1}{2} \frac{n-3}{2}\left(\frac{1}{r^{2}}-\frac{1}{\sinh ^{2} r}\right)$

The rest of the proof of (3.3) takes place in the hyperbolic setting exclusively. We may therefore drop the index hyp.

Region 2: Let us analyze the right hand side of (3.3), assuming that $t$ is bounded below and that $r=\mathcal{O}(t)$, let say $t \geq \max \left\{1, \frac{r}{2}\right\}$.

In this range, (3.3) amounts to

$$
h_{t}(r) \asymp t^{-\frac{3}{2}} \varphi_{0}(r) e^{-\left(\frac{n-1}{2}\right)^{2} t-\frac{r^{2}}{4 t}} .
$$

Here we have used (3.2), which reads

$$
\varphi_{0}(r) \asymp(1+r) e^{-\frac{n-1}{2} r}
$$

in the case $X=\mathrm{H}^{n}(\mathbb{R})$. We shall need another estimate for the basic spherical function $\varphi_{0}$, which played already a key role in $[\mathrm{ABJ}]$.

Lemma : $r \frac{d}{d r} \log \left(\delta^{\frac{1}{2}} \varphi_{0}\right)(r)=1+\mathcal{O}\left(\frac{1}{1+r}\right) \quad \forall r \geq 0$. 
Let us sketch a proof, for the sake of completeness. Since

$$
r \frac{d}{d r} \log \left(\delta^{\frac{1}{2}} \varphi_{0}\right)(r)=\frac{n-1}{2} r \operatorname{coth} r+r \frac{\varphi_{0}^{\prime}(r)}{\varphi_{0}(r)}
$$

is smooth, the problem lies at infinity. In order to handle it, let us recall Harish-Chandra's expansion

$$
2^{\frac{n-1}{2}} \delta(r)^{\frac{1}{2}} \varphi_{\lambda}(r)=\mathbf{c}(\lambda) \Phi_{\lambda}(r)+\mathbf{c}(-\lambda) \Phi_{-\lambda}(r)
$$

for spherical functions, which holds for $r>0$ and for generic $\lambda \in \mathbb{C}$, in particular for $\lambda \in \mathbb{R}^{*}$ (but not for $\lambda=0$ ). Here

and

$$
\mathbf{c}(\lambda)=\frac{\Gamma\left(\frac{n-1}{2}\right)}{\Gamma(n-1)} \frac{\Gamma(i \lambda)}{\Gamma\left(i \lambda+\frac{n-1}{2}\right)}
$$

$$
\Phi_{\lambda}(r)=\sum_{j=0}^{+\infty} \gamma_{j}(\lambda) e^{(i \lambda-2 j) r}
$$

is a converging series for $r>0$ and for $\lambda \in \mathbb{R}$, with $\gamma_{0} \equiv 1$. In rank one, this is actually part of special function theory, specifically Jacobi function theory (see for instance [Ko2]). In the limit case $\lambda=0$, we obtain the converging expansion

$$
\delta(r)^{\frac{1}{2}} \varphi_{0}(r)=\sum_{j=0}^{+\infty}\left(a_{j} r+b_{j}\right) e^{-2 j r} \quad \forall r>0,
$$

with $a_{0}>0$, after multiplying (3.9) by $\lambda$ and applying $\left.\frac{\partial}{\partial \lambda}\right|_{\lambda=0}$. Consequently

$$
\frac{d}{d r} \log \left(\delta^{\frac{1}{2}} \varphi_{0}\right)(r)=\frac{\frac{d}{d r}\left\{\delta(r)^{1 / 2} \varphi_{0}(r)\right\}}{\delta(r)^{1 / 2} \varphi_{0}(r)}=\frac{1}{r}+\mathcal{O}\left(\frac{1}{r^{2}}\right) \quad \text { as } r \rightarrow+\infty .
$$

This concludes the proof of the lemma.

Let us turn to the analysis of the right hand side $h_{2}(r, t)$ of $(3.7)$ in Region 2 . We get

$$
\frac{\mathrm{D} h_{2}}{h_{2}}=\frac{1}{t}\left\{r \frac{\partial}{\partial r} \log \left(\delta^{\frac{1}{2}} \varphi_{0}\right)(r)-1\right\}=\mathcal{O}\left(\frac{1}{t(1+r)}\right)
$$

by the lemma. Let us replace $h_{2}$ by

$$
h_{2}^{ \pm}(r, t)=e^{\mp c \frac{\sqrt{1+r^{2}}}{t}} h_{2}(t, r)
$$

where the sign and the size of the constant $c>0$ will be chosen appropriately. This modification is harmless for the right hand side of (3.3) and it will improve (3.10). Indeed, let us compute $\mathrm{D} h \frac{ \pm}{2} / h_{2}^{ \pm}=I+I I$, where

and

$$
I=\frac{1}{t}\left\{r \frac{\partial}{\partial r} \log \left(\delta^{\frac{1}{2}} \varphi_{0}\right)(r)-1\right\} \pm \frac{c}{t \sqrt{1+r^{2}}}\left\{2 r \frac{\partial}{\partial r} \log \left(\delta^{\frac{1}{2}} \varphi_{0}\right)(r)+\frac{1}{1+r^{2}}\right\}
$$

$$
I I= \pm \frac{c}{t^{2} \sqrt{1+r^{2}}}-\frac{c^{2} r^{2}}{t^{2}\left(1+r^{2}\right)}
$$

According to the lemma, the expression $I$ has the same sign as \pm , provided $c$ is large enough. Once $c$ is fixed, the expression $I I$ is $\mathcal{O}\left(\frac{1}{t^{2}}\right)$.

Region $\mathbf{3}=\mathbf{1}+\mathbf{2}$ : Let us establish (3.3), assuming that $t$ is bounded below, let say $t \geq 1$.

Let $\chi: \mathbb{R} \rightarrow[0,1]$ be a smooth cut off function such that

$$
\begin{cases}\chi \equiv 1 & \text { on }(-\infty, 1] \\ \chi \equiv 0 & \text { on }[2,+\infty)\end{cases}
$$

and set $h_{3}^{ \pm}(r, t)=\left\{1-\chi\left(\frac{r}{t}\right)\right\} h_{1}(r, t)+\chi\left(\frac{r}{t}\right) h_{2}^{ \pm}(r, t)$. Notice that the expressions $h_{1}(r, t), h_{2}(r, t), h_{2}^{ \pm}(r, t), h_{3}^{ \pm}(r, t)$ are all comparable when $r \asymp t$. Let us compute

$$
\begin{aligned}
\mathrm{D} h_{3}^{ \pm}(r, t) & =\left\{1-\chi\left(\frac{r}{t}\right)\right\} \mathrm{D} h_{1}(r, t)+\chi\left(\frac{r}{t}\right) \mathrm{D} h_{2}^{ \pm}(r, t) \\
& +\chi^{\prime}\left(\frac{r}{t}\right)\left\{\frac{n-1}{t r} h_{1}(r, t)-\frac{2}{t} \frac{\partial}{\partial r} \log \left(\delta^{\frac{1}{2}} \varphi_{0}\right)(r) h_{2}^{ \pm} \pm \frac{2 c r}{t^{2} \sqrt{1+r^{2}}} h_{2}^{ \pm}\right\} \\
& +\chi^{\prime \prime}\left(\frac{r}{t}\right) \frac{1}{t^{2}}\left\{h_{1}(r, t)-h_{2}^{ \pm}(r, t)\right\}
\end{aligned}
$$

It follows from previous considerations that all expressions occuring in $\mathrm{D} h \frac{ \pm}{3} / h_{3}^{ \pm}$either have the same sign as \pm or are $\mathcal{O}\left(\frac{1}{t^{2}}\right)$. Let us come to the upper heat kernel estimate. Fix temporarily $R>0$ and $T>1$. As for small $t$, we consider the heat kernel $h_{R, t}(r)$ in the ball $\Omega_{R}$, with Dirichlet boundary condition $h_{R, t}(R)=0$, and apply the minimum principle in $\Omega_{R} \times[1, T]$ to the function 


$$
u(r, t)=C e^{-\frac{c^{\prime}}{t}} h_{3}^{+}(r, t)-h_{R, t}(r) .
$$

Here $C$ and $c^{\prime}$ are appropriate positive constants, which depend neither on $R$ nor on $T$. First,

$$
\frac{\mathrm{D} u}{e^{-c^{\prime} / t} h_{3}^{+}}=\frac{c^{\prime}}{t^{2}}+\frac{\mathrm{D} h_{3}^{+}}{h_{3}^{+}}
$$

turns positive by choosing $c^{\prime}$ sufficiently large. Secondly, since (3.3) holds for small time,

$$
u(r, 1)=C e^{-c^{\prime}} h_{3}^{+}(r, 1)-h_{R, 1}(r) \geq C e^{-c^{\prime}} h_{3}^{+}(r, 1)-h_{1}(r)
$$

is positive provided $C$ is large enough. Thirdly,

$$
u(R, t)=C e^{-c^{\prime} / t} h_{3}^{+}(R, t)
$$

is obviously positive. It follows from the minimum principle that

$$
u(r, t) \geq 0 \quad \text { i.e. } \quad h_{R, t}(r) \leq C e^{-c^{\prime} / t} h_{3}^{+}(r, t)
$$

throughout $\Omega_{R} \times[1, T]$. Letting $R \nearrow+\infty$ and $T \nearrow+\infty$, we conclude that

$$
h_{t}(r) \leq C h_{3}^{+}(r, t) \quad \forall(r, t) \in[0,+\infty) \times[1,+\infty) .
$$

Let us turn to the lower estimate. We apply now the minimum principle in $\Omega_{R} \times[1, T]$ to

$$
u(r, t)=h_{t}(r)-C e^{\frac{c^{\prime}}{t}} h_{3}^{-}(r, t) .
$$

Arguying as before, we choose first $c^{\prime}>0$ large enough and next $C>0$ small enough so that

$$
\frac{\mathrm{D} u}{C e^{c^{\prime} / t} h_{3}^{-}}=\frac{c^{\prime}}{t^{2}}-\frac{\mathrm{D} h_{3}^{-}}{h_{3}^{-}} \quad \text { and } \quad u(r, 1)=h_{1}(r)-C e^{c^{\prime}} h_{3}^{-}(r, 1)
$$

are positive. This can be achieved independently of $R>2 T>2$. Moreover,

$$
u(R, t)=h_{t}(R)-C e^{\frac{c^{\prime}}{t}} h_{1}(R, t) \geq-C^{\prime} J(R)^{-\frac{1}{2}},
$$

where $C^{\prime}=C e^{c^{\prime}}(4 \pi)^{-n / 2}$. It follows from the minimum principle that

$$
u(r, t)+C^{\prime} J(R)^{-1 / 2} \geq 0
$$

throughout $\Omega_{R} \times[1, T]$. Letting $R \nearrow+\infty$ and $T \nearrow+\infty$, we get $u(r, t) \geq 0$ hence

$$
h_{t}(r) \geq C h_{3}^{-}(r, t) \quad \forall(r, t) \in[0,+\infty) \times[1,+\infty) .
$$

This concludes the proof of (3.3).

Our proof works the same in higher rank. It is just technically more involved. One deals separately with the cases $t \leq 1$ and $t \geq 1$, the first one being easiest. In the case $t \geq 1$, we split $\overline{\mathfrak{a}^{+}}$according to Arthur into $2^{\ell}$ regions depending on $t$, estimate the heat kernel in each region and glue the bounds together using a smooth partition of unity.

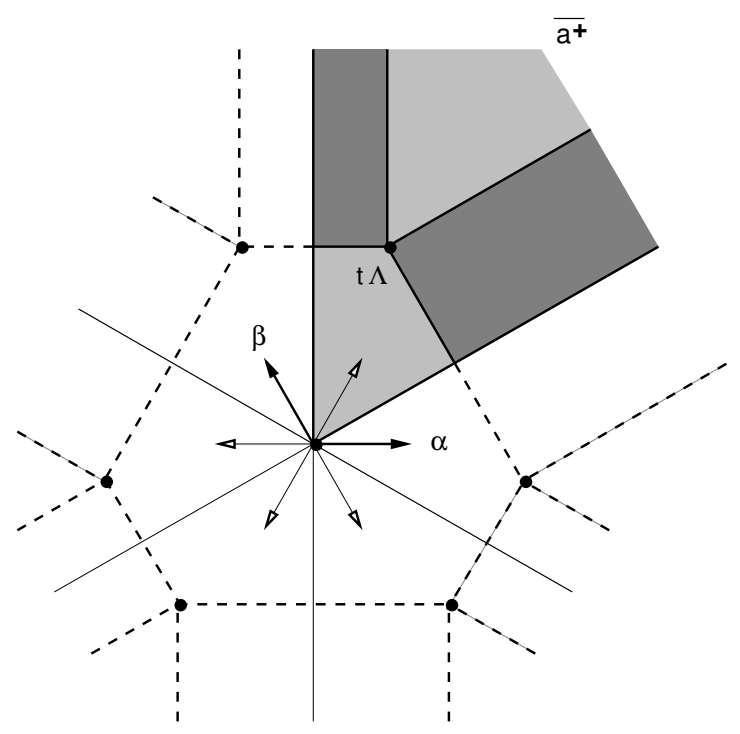


Compared to the previous approach in [AJ1], which consisted in analyzing carefully the inverse spherical transform (1.6), the present approach is more elementary and more flexible - it is likely to apply to other settings as well. On the other hand, to our knowledge it is limited to heat kernel bounds and yields neither asymptotics nor estimates of derivatives.

\section{Related REsults}

In this section we review some results which are connected with our main theorem. Most of them relied on previous heat kernel estimates and may be obtained more directly using the present ones.

\subsection{Heat propagation.}

The heat kernel $h_{t}(x)$ on $X$ concentrates asymptotically in an annulus

$$
\{x \in X|2| \varrho|t-r(t) \leq| x|\leq 2| \varrho \mid t+r(t)\}
$$

of width $r(t)$, growing any faster than $\sqrt{t}$. In higher rank, it concentrates more precisely in the bi- $K$-orbit of the ball $\mathrm{B}(2 t \varrho, r(t))$ in $\mathfrak{a}$ :

$$
\int_{K\{\operatorname{exp~B}(2 t \varrho, r(t))\} K} d x h_{t}(x) \rightarrow 1 \quad \text { as } t \rightarrow+\infty .
$$

This striking non Euclidean phenomenon has been observed by several authors. From a probabilistic point of view (see $[\mathrm{ABJ}]$ and notably $[\mathrm{B}]$ among the references cited therein), it follows from a central limit theorem for the radial part $H_{t} \in \overline{\mathfrak{a}^{+}}$of the Brownian motion in the Cartan decomposition. Specifically, $\left(H_{t}-2 t \varrho\right) / \sqrt{t}$ converges in distribution to a centered normal law. From an analytic point of view (see [AS]), (4.1) follows from sharp upper heat kernel estimates such as (3.1) and reflects a balance between the volume growth on one hand and the heat kernel decay on the other hand. One way to make this interplay more visible is to consider the $L^{p}$ counterpart of (4.1) that we recall from [AJ1]. The asymptotic behavior remains the same as long as $1 \leq p<2$ :

$$
\int_{K\{\operatorname{exp~B}(4(1 / p-1 / 2) t \varrho, r(t))\} K} d x h_{t}(x) \sim\left\|h_{t}\right\|_{L^{p}} .
$$

It is similar to the Euclidean case when $p=2$ :

$$
\left\{\int_{r_{1}(t) \leq|x| \leq r_{2}(t)} d x h_{t}(x)^{2}\right\}^{1 / 2} \sim\left\|h_{t}\right\|_{L^{2}}
$$

if $r_{1}(t) / \sqrt{t} \rightarrow 0$ and if $r_{2}(t) / \sqrt{t} \rightarrow+\infty$. Finally the heat kernel concentrates around the origin when $2<p \leq+\infty$ :

$$
\left\|h_{t}\right\|_{L^{p}(\mathrm{~B}(e K, r(t)))} \sim\left\|h_{t}\right\|_{L^{p}}
$$

provided $r(t) \rightarrow+\infty$. Let us specify that the symbol $\sim$ between two positive expressions means that their ratio tends to 1.

\subsection{Green function}

The Green function

$$
g_{\zeta}(x)=\int_{0}^{+\infty} d t e^{\left(|\varrho|^{2}-\zeta^{2}\right) t} h_{t}(x)
$$

is the convolution kernel of the resolvent

$$
\left(-\Delta-|\varrho|^{2}+\zeta^{2}\right)^{-1}=\int_{0}^{+\infty} d t e^{\left(|\varrho|^{2}-\zeta^{2}\right) t} e^{t \Delta} .
$$

According to (3.1), the subordination formula (4.2) makes sense for every $\zeta \in[0,+\infty)$ and yields the following estimates: 


$$
\begin{aligned}
& g_{\zeta}(x) \asymp\left\{\begin{array}{ll}
|x|^{2-n} & \text { if } n \geq 3 \\
\log \frac{1}{|x|} & \text { if } n=2
\end{array} \quad\right. \text { near the origin, } \\
& g_{\zeta}(x) \asymp\left\{\begin{array}{ll}
|x|^{\frac{1-\ell}{2}-\left|\Sigma_{0}^{+}\right|} \varphi_{0}(x) e^{-\zeta|x|} & \text { if } \zeta>0 \\
|x|^{2-\ell-2\left|\Sigma_{0}^{+}\right|} \varphi_{0}(x) & \text { if } \zeta=0
\end{array}\right. \text { away from the origin. }
\end{aligned}
$$

Similar results, as well as asymptotics, hold more generally for the kernels of fractional powers $\left(-\Delta-|\varrho|^{2}+\zeta^{2}\right)^{-\sigma / 2}($ see $[\mathrm{AJ} 1])$.

\subsection{Martin compactification.}

The behavior at infinity of the Green function $g_{\zeta}(x)$ plays a key role in the determination of the Martin compactification $\bar{X}_{\zeta}^{\mathrm{M}}$ of $X=G / K$, at least when $\zeta>0$. Recall that $\bar{X}_{\zeta}^{\mathrm{M}}$ comes from potential theory and consists of positive solutions to

$$
\left(-\Delta-|\varrho|^{2}+\zeta^{2}\right) u=0 .
$$

More precisely, interior points $y K \in G / K$ are identified with functions

$$
x K \longmapsto k_{\zeta}(x K, y K)=\frac{g_{\zeta}\left(y^{-1} x\right)}{g_{\zeta}\left(y^{-1}\right)}
$$

and boundary points are all possible limits of the Martin kernel $k_{\zeta}(., y K)$ as $|y| \rightarrow+\infty$. Dynkin [D] initiated the investigation of the Martin compactification of symmetric spaces with the example $G=\mathrm{SL}(n, \mathbb{C})$. Extremal points of $\partial_{\zeta}^{\mathrm{M}} X$, which constitute the socalled minimal Martin boundary, were determined by Karpelevič [Ka]. After a failed attempt by Olshanetsky (see [Ol1] for the announcement and [Ol2] for details), the full Martin compactification $\bar{X}_{\zeta}^{\mathrm{M}}$ was eventually determined by Guivarc'h, Ji \& Taylor ([GJT], $[\mathrm{Gu}]$ ). Let us recall their main result :

(i) Assume that $\zeta=0$. Then $\bar{X}_{\zeta}^{\mathrm{M}}$ coincides with the maximal Furstenberg-Satake compactification $\bar{X}^{\mathrm{FS}}$ of $X$.

(ii) Assume that $\zeta>0$. Then $\bar{X}_{\zeta}^{\mathrm{M}}$ is the smallest compactification of $X$ which dominates both $\bar{X}^{\mathrm{FS}}$ and the geometric compactification $\bar{X}^{\text {geom }}$ of $X$ by means of geodesic rays (also called conic or visual compactification). In other words, $\bar{X}_{\zeta}^{\mathrm{M}}$ coincides with the closure of $X$ diagonally embedded into $\bar{X}^{\mathrm{FS}} \times \bar{X}^{\text {geom }}$.

\subsection{Stable processes.}

The Poisson semigroup $e^{-t \sqrt{-\Delta}}$ is subordinated to the heat semigroup $e^{t \Delta}$ by

$$
e^{-t \sqrt{-\Delta}}=\frac{t}{2 \sqrt{\pi}} \int_{0}^{+\infty} d s s^{-\frac{3}{2}} e^{-\frac{t^{2}}{4 s}} e^{s \Delta} .
$$

The corresponding formula at the kernel level yields the following global bounds

$$
p_{t}(x) \asymp\left\{\begin{array}{cl}
t(t+|x|)^{-n-1} & \text { if } t+|x| \leq 1 \\
t(t+|x|)^{-\frac{\ell}{2}-\left|\Sigma_{0}^{+}\right|-1} \varphi_{0}(x) e^{-|\varrho| \sqrt{t^{2}+|x|^{2}}} & \text { if } t+|x| \geq 1
\end{array}\right.
$$

for the Poisson kernel, as well as full asymptotics when $t+|x| \rightarrow \infty$ (see [AJ1]). More generally one may consider the family of semigroups $t \longmapsto e^{-t(-\Delta)^{\alpha / 2}}$ with $0<\alpha<2$. They are still subordinated to the heat semigroup, but the formula is no more explicit. Graczyk and Stós [GS] have nevertheless managed to establish global bounds similar to the above ones. 


\subsection{Heat kernel estimates for a distinguished Laplacian on $N A$.}

According to the Iwasawa decomposition $G=N A K$, the symmetric space $X=G / K$ can be identified with the solvable Lie group $S=N A=A N$. The Laplace-Beltrami operator on $X$ writes

$$
\Delta f(x)=f\left(x: \sum_{j} H_{j}^{2}-2 \varrho+2 \sum_{k} X_{k}^{2}\right)
$$

on $S$, where $\left(H_{j}\right)$ and $\left(X_{k}\right)$ are orthonormal bases of $\mathfrak{a}$ and $\mathfrak{n}$ respectively. It is closely connected with the following Laplacian on $S$

$$
\mathrm{L} f(x)=\left\{a^{-\varrho} \circ\left(\Delta+|\varrho|^{2}\right) \circ a^{\varrho}\right\} f(x)=f\left(x: \sum_{j} H_{j}^{2}+2 \sum_{k} X_{k}^{2}\right),
$$

where $a^{ \pm \varrho}$ denotes the multiplicative character $x \mapsto a(x)^{ \pm \varrho}=e^{ \pm\langle\varrho, A(x)\rangle}$ of $S$. This operator has been considered by several authors, with a possible side exchange (see for instance [GM], [AJ1], [HS] and the references cited therein). Here it is left-invariant and self-adjoint with respect to the right-invariant Haar measure $d_{r} x=d n d a=e^{2} \varrho d a d n$. Notice that $a^{\varrho}$ is a ground state of $\Delta$ and that $\mathrm{L}$ is the generator of the associated relativized process. The corresponding heat kernels are related by

$$
h_{t}^{S}(x)=e^{|\varrho|^{2} t} a(x)^{-\varrho} h_{t}^{X}(x K) \quad \forall t>0, \forall x \in S .
$$

By transferring the estimate (3.1) for $h_{t}^{X}$, one gets

$$
h_{t}^{S}(x) \asymp t^{-\frac{\ell}{2}-\left|\Sigma_{0}^{+}\right|} a(x)^{-\varrho} \varphi_{0}(x) \omega(x, t) e^{-\frac{|x|^{2}}{4 t}} \quad \forall t>0, \forall x \in S,
$$

where $\omega(x, t)=\prod_{\alpha \in \Sigma_{0}^{+}}\left(1+\frac{\langle\alpha, H\rangle}{t}\right)^{\frac{m_{\alpha}+m_{2 \alpha}}{2}-1}$ if $x=k_{1} e^{H} k_{2}$ in the Cartan decomposition. Asymptotics, as well as estimates of derivatives, can be deduced similarly from [AJ1], provided $|x|=\mathcal{O}(1+t)$.

\subsection{Heat kernel estimates for subLaplacians on $G$.}

Instead of a specific Laplacian, consider now a general left-invariant subLaplacian

$$
\mathrm{L} f(x)=f\left(x: \sum_{j} X_{j}^{2}\right)
$$

on $G$. The only requirement is Hörmander's condition, stipulating that the $X_{j}$ 's generate $\mathfrak{g}$ as a Lie algebra. In this general setting, Lohoué and Alexopoulos [LA] have recently established the following heat kernel estimate (their restriction $|x|=\mathcal{O}(\sqrt{t})$ for the lower estimate can be easily removed $[\mathrm{AG}])$. Assume that $t$ is bounded below, let say $t \geq 1$. Then there exist positive constants $C_{1}, c_{1}, C_{2}, c_{2}$ such that

$$
C_{1} t^{-\frac{\ell}{2}-\left|\Sigma_{0}^{+}\right|} e^{-\sigma t} \varphi_{0}(x) e^{-c_{1} \frac{\|x\|^{2}}{t}} \leq h_{t}^{G}(x) \leq C_{2} t^{-\frac{\ell}{2}-\left|\Sigma_{0}^{+}\right|} e^{-\sigma t} \varphi_{0}(x) e^{-c_{2} \frac{\|x\|^{2}}{t}}
$$

for every $x \in G$. Here $\sigma>0$ denotes the spectral gap and $\|$. $\|$ the Carnot-Carathéodory distance to the origin. It is remarkable that this estimate is almost uniform in $\mathrm{L}$. It depends indeed only on the spectral gap and not on the actual distance considered, since all reasonable left-invariant distances are comparable at large scale (see for instance Proposition III.4.2 in [VSC]).

The estimate (4.4) can be sharpened for a class of subLaplacians resembling the LaplaceBeltrami operator $\Delta$ on $X=G / K$. More precisely, assume that

$$
\mathrm{L} f(x)=f\left(x: \Omega_{\mathfrak{p}}+\sum_{k} Z_{k}^{2}\right)
$$

where $\left(Y_{j}\right)$ is an orthonormal basis of $\mathfrak{p}$, so that $\Omega_{\mathfrak{p}}=\sum_{j} Y_{j}^{2}$ is the $\mathfrak{p}$-component of the Casimir element $\Omega$, and $\left(Z_{k}\right)$ is any family in $\mathfrak{k}$. For instance, $\operatorname{L} f(x)=f\left(x: \Omega_{\mathfrak{p}}\right)$ or $\mathrm{L} f(x)=f\left(x: \Omega_{\mathfrak{p}}+\Omega_{\mathfrak{k}}\right)$. Then, following a suggestion of Lohoué, the second author shows in [Os] that

(i) L and $\Delta$ have the same spectral gap: $\sigma=|\varrho|^{2}$,

(ii) the Carnot-Carathéodory distance $\|$.$\| is equal to |$.$| , up to an additive constant:$

$$
|x| \leq\|x\| \leq|x|+c \quad \forall x \in G \text {. }
$$


Moreover, if $t$ is bounded below, let say $t \geq 1$,

(iii) there exists a constant $C>0$ such that

$$
C^{-1} h_{t-\frac{1}{4}}^{X}(x) \leq h_{t}^{G}(x) \leq C h_{t+\frac{1}{4}}^{X}(x) \quad \forall x \in G .
$$

By using the estimate (3.1) for $h_{t \pm 1 / 4}^{X}(x)$, one gets

$$
\begin{aligned}
& C_{1} t^{-\frac{\ell}{2}-\left|\Sigma_{0}^{+}\right|} e^{-|\varrho|^{2} t} \varphi_{0}(x) \omega(x, t) e^{-\frac{\|x\|^{2}}{4(t-1 / 2)}} \leq \\
& \leq h_{t}^{G}(x) \leq C_{2} t^{-\frac{\ell}{2}-\left|\Sigma_{0}^{+}\right|} e^{-|\varrho|^{2} t} \varphi_{0}(x) \omega(x, t) e^{-\frac{\|x\|^{2}}{4(t+1 / 2)}}
\end{aligned}
$$

for every $t \geq 1$ and $x \in G$. Here $C_{1}$ and $C_{2}$ are positive constants and $\omega(x, t)$ has the same meaning as in (4.3). In particular,

$$
h_{t}(x) \asymp t^{-\frac{\ell}{2}-\left|\Sigma_{0}^{+}\right|} e^{-|\varrho|^{2} t} \varphi_{0}(x) e^{-\frac{\|x\|^{2}}{4 t}}
$$

if $t \geq 1$ and $|x|=\mathcal{O}(t)$

Remark : For lack of space and competence, we have not been able to cover several topics such as probabilistic aspects of heat diffusion on symmetric spaces (see [ABJ] and the references therein), heat kernels on Lie groups with exponential growth [VM] or on Cartan-Hadamard manifolds, and discrete analogs (random walks as far as processes are concerned, trees or buildings as far as spaces are concerned).

\section{REFERENCES}

[A1] J.-Ph. Anker, La forme exacte de l'estimation fondamentale de Harish-Chandra, C. R. Acad. Sci. Paris Sér. I (Math.) 305 (1987), 371-374.

[A2] Le noyau de la chaleur sur les espaces symétriques $\mathrm{U}(p, q) / \mathrm{U}(p) \times \mathrm{U}(q)$, in Harmonic analysis; Luxembourg 1987, P. Eymard \& J.-P. Pier (eds.), Lect. Notes Math. 1359, SpringerVerlag (1988), 60-82.

[ABJ] J.-Ph. Anker, Ph. Bougerol \& T. Jeulin, The infinite Brownian loop on a symmetric space, Rev. Mat. Iberoamericana 18 (2002), 41-97.

[ADY] J.-Ph. Anker, E. Damek \& C. Yacoub, Spherical analysis on harmonic AN groups, Ann. Scuola Norm. Sup. Pisa XXIII (1996), 643-679.

[AG] J.-Ph. Anker \& A. Grigor'yan, Note on a paper by Alexopoulos 6 Lohoué, In preparation.

[AJ1] J.-Ph. Anker \& L. Ji, Heat kernel and Green function estimates on noncompact symmetric spaces, Geom. Funct. Anal. (GAFA) 9 (1999), 1035-1091.

[AJ2] Heat kernel and Green function estimates on noncompact symmetric spaces II, in Topics in probability and Lie groups; boundary theory, J. C. Taylor (ed.), CRM Proc. Lect. Notes 28, Amer. Math. Soc. (2001), 1-9.

[AO] J.-Ph. Anker \& P. Ostellari, Global heat kernel bounds on noncompact symmetric spaces, In preparation.

[AS] J.-Ph. Anker \& A. G. Setti, Asymptotic finite propagation speed for heat diffusion on certain Riemannian manifolds, J. Funct. Anal. 103 (1992), 50-61.

[B] M. Babillot, A probabilistic approach to heat diffusion on symmetric spaces, J. Theoretical Prob. 7 (1994), 599-607.

[BK] F. A. Berezin \& F. I. Karpelevič, Zonal spherical functions and Laplace operators on some symmetric spaces, Dokl. Akad. Nauk SSSR 118 (1958), 9-12.

[C] I. Chavel, Eigenvalues in Riemannian geometry, Academic Press (1984).

[DM] E. B. Davies \& N. Mandouvalos, Heat kernel bounds on hyperbolic space and Kleinian groups, Proc. Lond. Math. Soc. 57 (1988), 192-208.

[DGM] A. Debiard, B. Gaveau \& E. Mazet, Théorèmes de comparaison en géométrie riemannienne, Publ. RIMS Kyoto 12 (1976), 391-425.

[D] E. B. Dynkin, Brownian motion in certain symmetric spaces and nonnegative eigenfunctions of the Laplace-Beltrami operator, Amer. Math. Soc. Transl. Ser. 2, 72 (1968), 203-228.

[F] M. Flensted-Jensen, Spherical functions of a real semisimple Lie group. A method of reduction to the complex case, J. Funct. Anal. 30 (1978), 106-146.

[Ga] R. Gangolli, Asymptotic behaviour of spectra of compact quotients of certain symmetric spaces, Acta Math. 121 (1968), 151-192. 
[GV] R. A. Gangolli \& V. S. Varadarajan, Harmonic analysis of spherical functions on real reductive groups, Springer-Verlag (1988).

[GM] S. Giulini \& G. Mauceri, Analysis of a distinguished Laplacian on solvable Lie groups, Math. Nachr. 163 (1993), 151-162.

[Go] R. Godement, Introduction aux travaux de A. Selberg, Séminaire Bourbaki 144 (1956/57).

[GS] P. Graczyk \& A. Stós, Transition density estimates for stable processes on symmetric spaces, Preprint (2002).

[Gr] J.-C. Gruet, Semi-groupe du mouvement brownien hyperbolique, Stochastics and Stochastic Reports 56 (1996), 53-61.

[GK] S. G. Gindikin \& F. I. Karpelevič, Plancherel measure for symmetric Riemannian spaces of non-positive curvature, Dokl. Akad. Nauk SSSR 145 (1962), 252-255.

[Gu] Y. Guivarc'h, Compactifications of symmetric spaces and positive eigenfunctions of the Laplacian, in Topics in probability and Lie groups; boundary theory, J. C. Taylor (ed.), CRM Proc. Lect. Notes 28, Amer. Math. Soc. (2001), 69-116.

[GJT] Y. Guivarc'h, L. Ji \& J. C. Taylor, Compactifications of symmetric spaces, Progress Math. 156, Birkhäuser (1998).

[HS] W. Hebisch \& T. Steger, Multipliers and singular integrals on exponential growth groups, Preprint (2001).

[H1] S. Helgason, Differential geometry, Lie groups, and symmetric spaces, Academic Press (1978).

[H2] Groups and geometric analysis, Academic Press (1984).

[H3] Geometric analysis on symmetric spaces, Amer. Math. Soc. (1994).

[Ka] F. I. Karpelevič, The geometry of geodesics and the eigenfunctions of the Beltrami-Laplace operator on symmetric spaces, Trans. Moscow Math. Soc. 1965, Amer. Math. Soc. (1967), 51199.

[Kn] A. W. Knapp, Lie groups beyond an introduction, Progress Math. 140, Birkhäuser (1996).

[Ko1] T. H. Koornwinder, A new proof of a Paley-Wiener type theorem for the Jacobi transform, Ark. Mat. 13 (1975), 145-159.

[Ko2] - Jacobi functions and analysis on noncompact semisimple Lie groups, in Special functions; group theoretical aspects and applications, R. A. Askey \& al. (eds.), Reidel (1984), 1-85.

[LA] N. Lohoué \& G. K. Alexopoulos, On the large time behavior of heat kernels on Lie groups, Duke Math. J. (to appear).

[LRy] N. Lohoué \& Th. Rychener, Die Resolvente von $\Delta$ auf symmetrischen Räumen vom nichtkompakten Typ, Comment. Math. Helv. 57 (1982), 445-468.

[LRo] G. Lorang \& B. Roynette, Etude d'une fonctionnelle liée au pont de Bessel, Ann. Inst. Henri Poincaré Section B (Prob. Stat.) 32 (1996), 107-133.

[Mc] H. P. McKean, An upper bound to the spectrum of $\Delta$ on a manifold of negative curvature, J. Diff. Geom. 4 (1970), 359-368.

[O11] M. A. Olshanetsky, The Martin boundary for the Laplace-Beltrami operator on a Riemannian symmetric space of nonpositive curvature, Usp. Mat. Nauk. 24 (1969), 189-190.

[Ol2] - Martin boundaries for real semisimple Lie groups, J. Funct. Anal. 126 (1994), $169-216$.

[Os] P. Ostellari, Global behavior of the heat kernel associated with certain sub-Laplacians on semisimple Lie groups, J. Funct. Anal. 199 (2003), 521-534.

[PW] M. H. Protter \& H. F. Weinberger, Maximum principles in differential equations, Prentice-Hall \& Springer-Verlag (1967 \& 1984).

[S1] P. Sawyer, The heat equation on the spaces of positive definite matrices, Canad. J. Math. 44 (1992), 624-651.

[S2] $\quad$ On an upper bound for the heat kernel on $\mathrm{SU}^{*}(2 n) / \mathrm{Sp}(n)$, Canad. Math. Bull. 37 (1994), 408-418.

[S3] - Estimates for the heat kernel on $\operatorname{SL}(n, \mathbb{R}) / \mathrm{SO}(n)$, Canad. J. Math. 49 (1997), 359-372.

[S4] Survey on the Abel transform, To appear in this volume.

[VM] N. Th. Varopoulos \& S. Mustapha, Analysis on Lie Groups., To appear in London Math. Soc. Lect. Note Series, Cambridge Univ. Press.

[VSC] N. Th. Varopoulos, L. Saloff-Coste \& T. Coulhon, Analysis and geometry on groups, Cambridge Univ. Press (1992).

Jean-Philippe Anker, Université d'Orléans, Laboratoire de Mathématiques MAPMO (UMR 6628), B.P. 6759, F-45067 Orléans Cedex 2, France 
E-mail address: Jean-Philippe.Anker@labomath.univ-orleans.fr

Patrick Ostellari, Université Henri Poincaré (Nancy I), Institut de Mathématiques Elie Cartan (Laboratoire Commun UHP-CNRS-INRIA), B.P. 239, F-54506 VandoeuvreLÈs-NANCY CEDEX, FranCE

E-mail address: ostel@iecn.u-nancy.fr 